\title{
Hole-Pair Formation in Cuprate Superconductors despite Antiferromagnetic Fluctuations
}

\author{
Rana Janardon Singh', Shakeel Khan² \\ ${ }^{1}$ Physics Department, Aligarh Muslim University, Aligarh, India \\ ${ }^{2}$ Department of Applied Physics, Aligarh Muslim University, Aligarh, India \\ Email: ranajsingh@yahoo.com, skhanapd@gmail.com
}

Received 1 May 2014; revised 13 June 2014; accepted 28 June 2014

Copyright (C) 2014 by authors and Scientific Research Publishing Inc.

This work is licensed under the Creative Commons Attribution International License (CC BY). http://creativecommons.org/licenses/by/4.0/

cC) (i) Open Access

\section{Abstract}

We have earlier proposed models of preformed hole pairs based on the results of our electron paramagnetic resonance experiments. A hole doped in a cuprate superconductor causes ferromagnetic alignment of the spins of the holes of $4 \mathrm{Cu}^{2+}$ ions of the plaquette $(\mathrm{CuO})_{4}$ in which it enters. Spin alignments undergo oscillations from vertically upward to vertically downward of the $\mathrm{CuO}_{2}$ plane. Vertical projections of spins go on changing when they pass through different plaquettes going to zero when they pass through the $\mathrm{CuO}_{2}$ plane. Ferromagnetic alignments of spins produce magnetic fields on the plane proportional to their vertical projections. When two holes travelling in $\mathrm{CuO}_{2}$ plane come across each other at a certain distance between them, they are attracted towards each other by Heisenberg exchange interaction and their path is decided by the magnetic field produced due to spin alignments. Their path is similar to $3 d x^{2}-y^{2}$ atomic orbital. Y-123 has been chosen as an example. Due to plethora of evidence of antiferromagnetic fluctuations in cuprates, hole-pair formation has been tried in Y-123 assuming antiferromagnetic fluctuations in it. It has been found that hole-pair formation in spite of AFM fluctuations can be explained on the same lines as done earlier. Hole-pair formation was tried in Tl-2201 to test whether the same rules apply in cuprates with very high coherence lengths. Coherence length in Tl-2201 = 52 Å, whereas in $\mathrm{Y}-123=15-20 \AA \AA$ in $\mathrm{CuO}_{2}$ plane. It has been reported that in $\mathrm{Tl}-2201$ the $\mathrm{CuO}_{2}$ plane is very flat and smooth. From this it was concluded that high coherence length is the result of the smoothness of the plane. Further it was concluded that the smoothness of the $\mathrm{CuO}_{2}$ plane depends upon the nature of the near neighbors of the $\mathrm{CuO}_{2}$ plane. Near neighbors of $\mathrm{Y}-123$ and Tl-2201 have been compared.

\section{Keywords}

Hole-Pair Formation in Cuprate Superconductors, Buckling Angle in $\mathrm{CuO}_{2}$ Plane, Coherence 


\section{Length in a-b-Plane $(\xi a b)$}

\section{Introduction}

In the absence of consensus regarding the mechanism of superconductivity in cuprates, people are thinking of preformed hole-pairs [1] [2] which below a certain temperature undergo Bose-Einstein condensation and cause superconductivity. We have proposed models of preformed hole-pairs [3]-[5] for resistanceless current flow in the a-b plane and along c-axis of cuprate superconductors. For better understanding of this paper, it should be read in conjunction with the paper in Ref. [4] because the detailed treatment given in [4] cannot be reproduced here. However, some salient points of the paper [4] are given here which may be of help to readers. As prepared, cuprate superconductors are electron paramagnetic resonance (EPR) silent because of antiferromagnetic (AFM) coupling of $\mathrm{Cu}^{2+}$ ions in the all-important $\mathrm{CuO}_{2}$ plane. When cuprate superconductors are deoxygenated, they show EPR spectra of $\mathrm{Cu}$-monomer, dimmer, tetramer and octamer, but more frequently $(\mathrm{CuO})_{4}$ tetramer. $(\mathrm{CuO})_{4}$ tetramer is the unit cell of two-dimensional (2-D) $\mathrm{CuO}_{2}$ plane and by understanding its properties, one can understand the properties of $\mathrm{CuO}_{2}$ plane which is the seat of superconductivity. Spectra of $(\mathrm{CuO})_{4}$ and other fragments [6]-[13] given above are observed also in constituents of superconductors as $\mathrm{BaCuO}_{2}, \mathrm{SrCuO}_{2}, \mathrm{CaCuO}_{2}$, $\mathrm{Bi}_{2} \mathrm{CuO}_{4}, \mathrm{CuO}$ etc. On deoxygenation, $(\mathrm{CuO})_{4}$ unit gets magnetically isolated from the bulk by breaking $8 \mathrm{Cu}-\mathrm{O}$ bonds situated at its 4 corners. In [14], it has been found that $\mathrm{Cu}-\mathrm{O}$ bonds are $\approx 87.5 \%$ ionic and $\approx 12.5 \%$ covalent. Breaking of 8 bonds amounts to loss of one electron from the $(\mathrm{CuO})_{4}$ plaquette, because oxygen is more electronegative than copper. Loss of one electron means the introduction of one hole in the $(\mathrm{CuO})_{4}$ plaquette. This situation can be represented by the following equation.

An isolated $(\mathrm{CuO})_{4}$ unit $=(\mathrm{CuO})_{4}$ unit of continuous sheet of $\mathrm{CuO}_{2}$ plane + a hole inside it. Observation of 4 fine structure signals which corresponds to total electronic spin $=2$ and 13 components in each fine structure line which corresponds to total nuclear spin $I=6$ suggest that advent of a hole in a $(\mathrm{CuO})_{4}$ entity causes ferromagnetic (FM) coupling of spins of $4 \mathrm{Cu}^{2+}$ ions resulting into total electronic spin $=2$ and 13 hyperfine components in each fine structure signal suggest that the advent of the hole in $(\mathrm{CuO})_{4}$ entity causes 4 holes of the $4 \mathrm{Cu}^{2+}$ ions tocirculate around the $(\mathrm{CuO})_{4}$ framework and each hole comes into contact with all $4 \mathrm{Cu}^{2+}$ ions. As discussed above, on introduction of a hole in a $(\mathrm{CuO})_{4}$ plaquette, spins of all $4 \mathrm{Cu}^{2+}$ ions align ferromagnetically and should continue to fluctuate with the same frequency corresponding to $41 \mathrm{meV}$ antiferromagnetic signal seen in Y-123 which we have chosen as an example. $41 \mathrm{meV}$ equated to $\mathrm{h} v$ gives $v$ to be equal to $10^{13}$ hertz or time-period $T=10^{-13}$ sec. But the question will be in which direction? It has been noted in [1] that modest magnetic field when it is perpendicular to $\mathrm{CuO}_{2}$ plane suppresses AFM resonance in $\mathrm{YBa}_{2} \mathrm{Cu}_{3} \mathrm{O}_{6.6}$ more significantly than when applied horizontally. It suggests that the FM coupled spins of holes of $4 \mathrm{Cu}^{2+}$ ions fluctuate vertically above and below the $\mathrm{CuO}_{2}$ plane. Starting from a position where FM aligned spins stand vertically upward of the $\mathrm{CuO}_{2}$ plane, they have maximum resultant spin angular momentum. When they cross $\mathrm{CuO}_{2}$ plane, spins find themselves spread in $\mathrm{CuO}_{2}$ plane. The diagonally opposite ones directed in opposite directions with resultant spin angular momentum equal to zero after a time $=T / 4$. After next $T / 4$, they get $\mathrm{FM}$ aligned vertically downward of the $\mathrm{CuO}_{2}$ plane; after another $T / 4$, the yagain find themselves spread in $\mathrm{CuO}_{2}$ plane with zero spin angular momentum. Yet after another $T / 4$, they go back to the original positions or vertically aligned above the $\mathrm{CuO}_{2}$ plane. The alignment of spins of $\mathrm{Cu}^{2+}$ holes and their circulation around the $(\mathrm{CuO})_{4}$ plaquette produce magnetic field on the $\mathrm{CuO}_{2}$ plane in proportion to resultant spin angular momentum and locations of holes during their circulation. The magnetic field produced has been calculated [4] to vary from $\approx+5300 \rightarrow 0 \rightarrow-5300$ gauss.

When 2 holes wandering in the $\mathrm{CuO}_{2}$ sheet of a superconductor come across a column or row of $(\mathrm{CuO})_{4}$ plaquettes within a certain distance, they are attracted towards each other by Heisenberrg exchange interaction and follow a path guided by the exchange interaction between them and magnetic field generated by the directions of spins of $\mathrm{Cu}^{2+}$ holes and their locations during circulation around a $(\mathrm{CuO})_{4}$ plaquette in which they are situated at that instant. The path executed by the pair of holes is similar to $3 d_{x 2-y 2}$ atomic orbital which has been supported by many theories and experiments. The wandering hole hops from one oxygen ion to the other forcing the holes of $\mathrm{Cu}^{2+}$ ions to circulate along the periphery of the $(\mathrm{CuO})_{4}$ plaquette. The spin angular momentum of 
wandering hole does not combine with spin or angular momentum of $\mathrm{Cu}^{2+}$ holes. To avoid confusion, let it be clear that the holes wandering in the $\mathrm{CuO}_{2}$ plane due to doping will be called wandering holes and those associated with $\mathrm{Cu}^{2+}$ ions will be called $\mathrm{Cu}^{2+}$ holes.

\section{Formation of Hole-Pairs}

A brief description of hole-pair formation is given below. Mathematical treatment of hole-pair formation in cuprates has been given in [4]. The shape of the hole-pair has been shown in Figure 3 of [4]. This figure has been reproduced in this paper as Figure 1. Full mathematics is avoided here, but some portions are explained here which will be of help in grasping the main idea and final equation describing the order parameter. Application of the equation in describing the shape of the hole-pair has been shown through a shortened table which is a part of Table 1 in [4]. The shortened table is numbered 1 in this paper.

In Figure 1, the squares A, B, C, D, E, F, G. H. I are the unit cells of $\mathrm{CuO}_{2}$ 2-D plane, each taken to be a square of side $38.4 \AA$, which is one side of a plaqette in a representative Y-123 superconductor (though in actual case there is a very small difference between a and b sides). When 2 wandering holes enter Figure 1, Hole 1 from A side and Hole 2 from $E$ side, they move towards each other under the effect of Heisenberg exchange interaction. Their velocities are modified by the magnetic field present in each $(\mathrm{CuO})_{4}$ plaquette they traverse.

Here we will explain some symbols and terms and the final equation which determines the path of holes in the preformed hole-pairs. Table 1 in this paper which shows position of holes at different angles along their paths in Figure 1 will be discussed for further clarification.

The velocity of a charged particle (here hole) does not change by magnetic field; only its direction is changed and $V^{2}$ is always equal to $V_{x}^{2}+V_{y}^{2}$. Motion of charged particle of charge "e" in $X Y$-plane under the action of magnetic field $\mathrm{Hz}$ (in $\mathrm{z}$-direction) is given by the following equations:

$$
\begin{gathered}
\frac{\mathrm{d} V x}{\mathrm{~d} t}=\left(\frac{\mathrm{e}}{m c}\right) H_{z} V_{y} \\
\frac{\mathrm{d} V y}{\mathrm{~d} t}=-\left(\frac{\mathrm{e}}{m c}\right) H_{z} V_{x} \\
\frac{\mathrm{d} V z}{\mathrm{~d} t}=0
\end{gathered}
$$

where $m$ is the mass of the charged particle. Equation (3) goes to zero and need not be considered further.

In the above equations, the three quantities $H_{z}, V_{x}$ and $V_{y}$ have been used in the following forms:

$H_{z}=H_{z} \cos w t ; V_{x}=V_{x} \sin w t ; V_{y}=(-) V_{y} \cos w t$, because magnitude of these quantities depends on the positions of holes in the figure which depends on the angle $\omega t . V_{y}$ has been expressed as $V_{y} \cos w t$ with a negative sign, because in this problem, the origin has been chosen at the center of the cell $\mathrm{A}$ and for all positions of the holes except at the origin, $y$-coordinate is always negative.

For angular velocities also, two notations have been used: $\omega^{\prime}$ and $\omega . \omega^{\prime}$ corresponds to the Euclidean angle according to which the total angle in going round a circle is $=360^{\circ}$ or $=2 \pi$. But for the spins of $\mathrm{Cu}^{2+}$ holes, one oscillation is completed for Hole 1 in going from A to $\mathrm{E}$ and for Hole 2 in going from $\mathrm{E}$ to A. Both these angles are equal to $180^{\circ}$ according to Euclidean geometry. This is why on the circumference of the circle in Figure 1, both the angles, $\omega^{\prime}$ and $\omega$ have been shown. It means that $\omega^{\prime}=2 \omega$. With this much introduction, it will be easy to appreciate the full meaning of the final formula in Ref. [4] defining the positions of holes at different angles as shown in Figure 1. The final formula in [4] is shown below and in this paper, it is numbered 4.

$$
R \cdot \omega t= \pm \frac{1}{2} \cdot R \cdot \ln \left|\tan \left(\frac{\omega t}{2}+\frac{\pi}{4}\right)\right|
$$

In Equation (4), $R$ is some length used for plotting this equation. $R$ has been given a nominal value of $7.68 \AA$, which is twice the side (38.4 $\AA$ ) of the unit cell of 2-dimensional $\mathrm{CuO}_{2}$ plane of $\mathrm{Y}-123$ superconductor. The R.H.S. of Equation (4) indicates the position of a wandering hole at an angle $\omega t$. The magnitude of the R.H.S. of Equation (4) is shown by a straight line from the coordinate of the angle $\omega t$ on the circumference of 


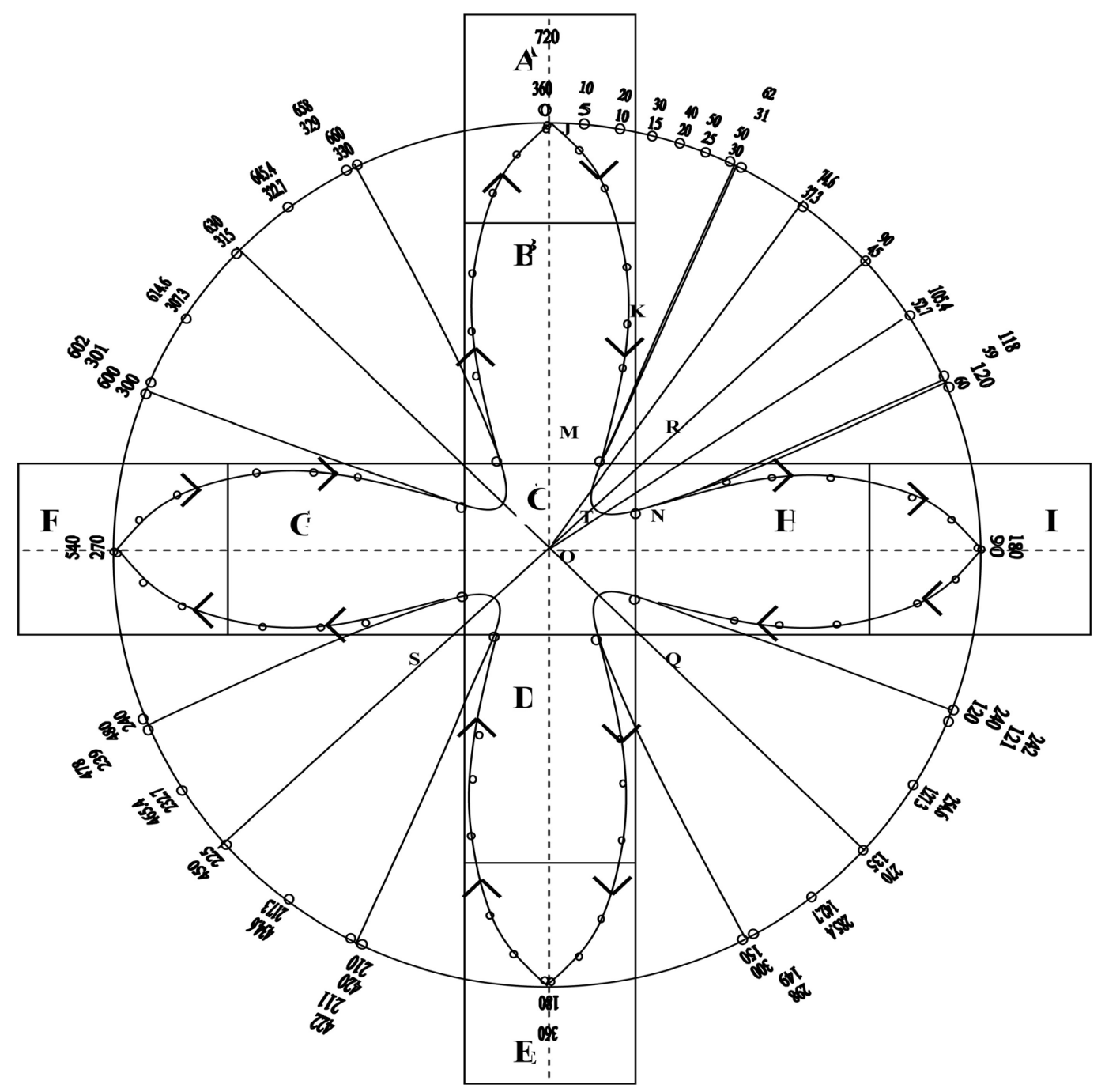

Figure 1. Formation of hole-pairs, both the holes traverse the same path continuously.

the circle towards the center of the circle. The tips at the end of lines (shown by dots) represent the positions of wandering holes. The magnitudes of the R.H.S. of Equation (4) for angles varying from $0^{\circ}$ to $360^{\circ}$ have been given in Table 1. In the range of angles $0^{\circ}$ to $180^{\circ}$ all the values are positive, but in the range $180^{\circ}$ to $360^{\circ}$ all the values are negative. But in the range of $180^{\circ}$ to $360^{\circ}$, the direction of the magnetic field is also negative, that is , downward of the $\mathrm{CuO}_{2}$ plane, whereas in the range $0^{\circ}$ to $180^{\circ}$, it is upward of the $\mathrm{CuO}_{2}$ plane. In Equation (4) also, there is \pm sign. It means in both ranges, the holes are attracted towards the center of the circle or the center of the cell C. With the help of Table 1 and Figure 1, meaning of the Equation (4) can be understood. So far discussion has been mainly for Hole 1. But same is true for Hole 2 which starts from $\mathrm{E}$ and completes $360^{\circ}$ of journey ( $360^{\circ}$ to $720^{\circ}$ ). In Figure 1 , Holes 1 and 2 always occupy positions diagrammatically opposite to each other.

Going through Table 1, one finds that at certain angles $(\omega t)$ the position of Hole 1 lies beyond the cell C. From $\omega t=80^{\circ}$ to $100^{\circ}$, the position of Hole 1 lies beyond the cell C. At $\omega t=90^{\circ}$, the position of Hole 1 is indeterminate according to Equation (4) or effectively very far from the cell C. At $\omega t=74.6^{\circ}$, the position of Hole 1 is just at the center of the cell C. The position of Hole 2 at $\omega t=74.6^{\circ}+360^{\circ}=434.6^{\circ}$ is also at the center of the cell C. Two holes cannot be present at the same place at the same time. Due to Coulomb repulsion, they cannot come very near to each other in the cell C.

Hole 1 remains inside the cell C within angles $\omega t=62^{\circ}$ to $118^{\circ}$. Similarly Hole 2 remains inside the cell 
Table 1. $\omega t=$ angle which the spin vector of the hole makes with a direction perpendicular to the $\mathrm{CuO}_{2}$ plane. In the rotation of $360^{\circ}$ in the Cartesian system, the spin vector completes two oscillations and so $\omega$ t varies from $0^{\circ}-720^{\circ}$ The expression $3.84 \cdot \ln \cdot\left|\tan \left(\frac{\omega t}{2}+\frac{\pi}{4}\right)\right|$ gives the position of the hole at the angle $\omega t$ in the (3).

\begin{tabular}{cccc}
\hline$t$ & $3.84 \cdot \ln \cdot\left|\tan \left(\frac{\omega t}{2}+\frac{\pi}{4}\right)\right|$ & $(1)$ & \\
$(1)$ & $(2)$ & & 0.000 \\
& 0.000 & 180 & -1.367 \\
0 & 1.367 & 200 & -2.927 \\
40 & 2.927 & 220 & -5.053 \\
60 & 5.053 & 240 & -5.333 \\
62 & 5.333 & 242 & -7.680 \\
74.6 & 7.680 & 254.6 & -9.354 \\
80 & 9.354 & 260 & -18.207 \\
89 & 18.207 & 269 & Indeterminate \\
90 & Indeterminate & -18.207 \\
91 & 18.207 & 270 & -9.354 \\
100 & 9.354 & 271 & -7.680 \\
105.4 & 7.680 & 280 & -5.333 \\
118 & 5.333 & 285.4 & -5.053 \\
120 & 5.053 & 298 & -2.927 \\
140 & 2.927 & 300 & -1.367 \\
160 & 1.367 & 320 & 0.000 \\
180 & 0.000 & 340 & \\
\hline
\end{tabular}

C within angles $\omega t=\left(360+62=422^{\circ}\right)$ to $\left(360+118=478^{\circ}\right)$. Inside the cell C, holes repel each other, their velocities are reduced and Heisenberg exchange interaction becomes ineffective due to such a small separation between the two charged particles. From the cell C, Hole 1 is deflected towards cells H-I and Hole 2 towards the cells G-F. When Hole 1 reaches cell I and Hole 2 reaches cell F, they experience maximum magnetic field because of vertical alignment of all the $4 \mathrm{Cu}^{2+}$ spins in respective cells. They are turned back from these cells due to magnetic mirror effect. Just to refresh memory, magnetic mirror effect is that force that causes the ions in the ionosphere to oscillate between the north pole and the south pole of the earth due to highest strength of the magnetic field at the two poles. The path of both the holes has been shown in Figure 1. The holes are indistinguishable and both holes follow the path A-C-I-C-E-C-F-C-A. Ultimately they circulate along a path similar to the shape of the atomic orbital $3 d_{x 2-y 2}$. Complete derivation of Equation (4) has been given in [4].

\section{Hole-Pair Formation in Spite of Antiferromagnetic Fluctuations}

It has been observed that in high $T_{c}$ cuprate superconductors [15]-[22] and in heavy Fermion systems $\left(\mathrm{UPd}_{2} \mathrm{Al}_{3}, \mathrm{CeCoIn}\right)_{5}$ [23] [24] and also in iron oxide superconductor $\left(\mathrm{Ba}_{0.6} \mathrm{~K}_{0.4} \mathrm{Fe}_{2} \mathrm{As}_{2}\right)$ [25], inelastic neutron scattering exhibit AFM fluctuations dominated by a resonance signal in single layered $\mathrm{CuO}_{2}$ superconductors [21] [22], there appears only one resonant signal; in two-layered superconductors [15]-[20], two signals are observed of odd and even parity where the modes differ in symmetry with respect to exchange between adjacent $\mathrm{CuO}_{2}$ layers. Odd parity signal which is resonance signal is stronger and occurs at smaller energy and the even parity signal is weaker and occurs at higher energy. A universal relation between AFM resonance signal and superconducting gap $(2 \Delta)$ has been demonstrated according to which energy of resonance signal $E_{r}$ is proportional to $2 \Delta$, but always less than $2 \Delta$ [26]. McDonald et al. [27] have pointed out that the experimentally determined Cooper pair wave function in cupratesmaps directly on the spin fluctuation disturbance responsible for the AFM peaks measured in inelastic neutron scattering. Large number of works cited above showing 
AFM excitations, commensurate or incommensurate suggest that these fluctuations are intrinsic property of cuprate superconductors and so should be integral part of any theory. But these fluctuations have failed to explain superconductivity.

\subsection{Degree of Antiferromagnetism and Ferromagnetism with Doping Level}

Kopp et al., [28] noted that any trace of AFM fluctuation is absent in over doped regime of cuprates. Superconductivity arises in Mott insulators after doping level of nearly 5\%; attains optimal value at 16\%; overdoping starts at $19 \%$ and the superconducting dome terminates in the vicinity of $25 \%$. In the overdoped regime, spin susceptibility shows a ferromagnetic upturn. He also suggested that at the end of superconductivity dome, there should be genuine ferromagnetism at zero temperature. Sonier et al. [29] observed gradual disappearance of antiferromagnetism on doping of cuprates and onset of static magnetic order in the highly doped regimes. But this magnetism is not of long range order but magnetic moments appear in dilute form. The main point in [28] [29] is that charge doping or hole-doping induces FM order in cuprates and competing ferromagnetic fluctuations are simultaneously present with superconductivity. It has also been concluded that on increasing doping level upto $25 \%$, ferromagnetic fluctuations increase at the expense of antiferromagnetic fluctuations.

\subsection{EPR Signals Due to Ferromagnetism though Experimentally Only Antiferromagnetic Fluctuations Are Observed}

We have proposed models of preformed hole-pairs in cuprates [3]-[5] on the basis of FM spin fluctuation in $(\mathrm{CuO})_{4}$ plaquette of $\mathrm{CuO}_{2}$ plane, but there is plethora of evidence that AFM fluctuations are intrinsic properties of cuprates. We will now show that hole-pairs can be formed on the same lines as done earlier without consideration of AFM fluctuations. For this, let us consider only A, B, C cells of Figure 1, shown separately in Figure 2.

We will consider the behaviour of Hole 1 only and the same applies to Hole 2. When the hole1 enters cell A of Figure 2, the spins of all the $4 \mathrm{Cu}^{2+}$ ions at its corners align vertically above the $\mathrm{CuO}_{2}$ plane as has been concluded from our EPR experiments and has been used in the formation of hole-pairs in Figure 1. It is to be remembered from Figure 1 that when a hole goes from cell A to B and then to C, the vertical components of spins gradually decrease and so also the magnetic field produced from them. Presently we are concerned with the states of spins 3, 4, 5, 6 when Hole 1 enters B from A. The spins 3 and 4 will remain in ferromagnetic alignment, but their spins will be tilted from the vertical direction in direct proportion to the fraction of time-period $\mathrm{T}$ of spin fluctuation spent by the hole in coming from A to B cell. But what about the spins numbered 5 and 6.Will there be FM exchange between the pair of spins 3 and 4 and the pair of 5 and 6 spins or AFM exchange. But because there is a hole present in the cell B [28] [29] which cause FM alignment of spins in any cell, so all the 4 spins 3, 4, 5, 6 will be in FM arrangement, but all 4 of them more inclined towards the horizontal plane. When the hole reaches the cell $\mathrm{C}$, there will be again realignment of spins with decreased vertical component. When the hole reaches the center of the cell $\mathrm{C}$, the total vertical component will be zero. As has been discussed in the hole-pair formation in Figure 1, the hole will never reach the center of the cell $\mathrm{C}$, because at the same time the other partner of the hole pair would also reach the center of the cell $\mathrm{C}$ which is not possible because of the Coulomb repulsion between the holes. Thus the whole process of hole-pair formation is in the same way as in the formation of hole-pair described in Figure 1.

But here a question arises, why in neutron diffraction experiments on cuprate superconductors, only AFM fluctuations are observed and not FM fluctuations. To answer this question, let us calculate the percentages of FM and AFM alignments of spins during one time-period $T$ of journey of Holes 1 and 2 in Figure 1. The calculation of FM and AFM order can be done under 2 assumptions: 1) in whichever 2 cells out of the 9 cells the 2 holes are present, they will be in FM alignment and the rest 7 in AFM alignment. In this way, during one time-period $T$ of journey of two holes in Figure $1,2 / 9 \times 100 \% \approx 22 \%$ will be in FM order and $\approx 78 \%$ in AFM order.

Under the assumption (2) when Holes 1 and 2 are in cells A and E respectively in the beginning, there will be FM order in cells A and E and in the rest 7, AFM order. When Hole 1 enters cell B and Hole 2 enters cell D, there will be subdued FM order in cells A and B due to Hole 1 and in cells E and D due to Hole 2, because there is common boundary between $\mathrm{A}$ and B for Hole 1 and common boundary between E and D for Hole 


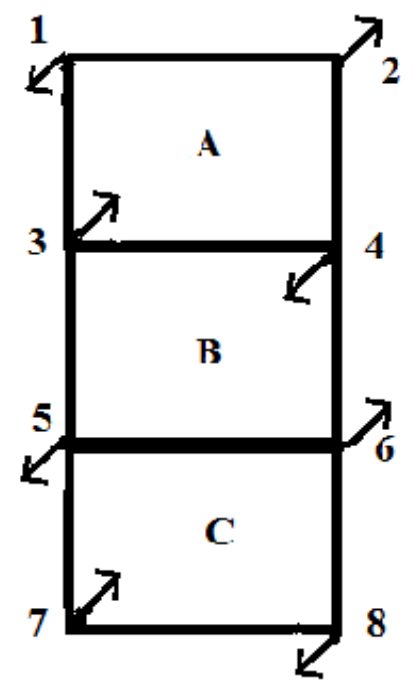

Figure 2. Showing spin configuration in AFM alignment. Spins have been numbered.

2. It can be said that there is half FM order both in A and B due to Hole 1 and half FM order both in D and E due to Hole 2. Half FM order is justified, because when both the holes enter cell C, FM order is nearly lost. It can be said that full ferromagnetism is due to the hole1 while in A and no ferromagnetism while in C. Thus when Hole 1 has reached cell B from A, it can be appropriately said that in both cells there is ferromagnetism of half strength only. From the cell C, due to curvature of the paths of holes and Coulomb repulsion, Hole 1 is reflected towards cells H-I and Hole 2 towards G-F. When Holes 1 and 2 reach cell H and G respectively, half FM order is attained, but FM order is built downward of the $\mathrm{CuO}_{2}$ plane. When Holes 1 and 2 reach I and F cells respectively, there will be full FM order downward of the $\mathrm{CuO}_{2}$ plane. When the holes turn back from the cells I and $\mathrm{F}$ at the extreme ends, they enter cells $\mathrm{H}$ and $\mathrm{G}$ respectively, half FM order is obtained in cells I and $\mathrm{H}$ due to Hole 1 and in cells $\mathrm{F}$ and $\mathrm{G}$ due to Hole 2. Again both the holes reach cell $\mathrm{C}$, where there will be negligible or zero magnetic order. From there, Hole 1 will be reflected towards cells D-E and Hole 2 towards cells B-A with the same type of magnetic order as in going from cell $\mathrm{C}$ towards cells $\mathrm{H}$-I for the Hole 1 and towards G-F for Hole 2, but FM order now building upward of the $\mathrm{CuO}_{2}$ plane. When Hole 1 has reached cell $\mathrm{E}$ and Hole 2 cell A, full FM order will be attained and by this time their journey in one time-period $\mathrm{T}$ has been completed. Thereafter they will be following the same path repeatedly.

Calculations by the first assumption gives $\approx 22$ percent of cells in FM spin alignment and $\approx 78$ percent in AFM alignment. Calculation by the second method gives 16\% FM order and $\approx 84 \%$ AFM order. Calculation of FM and AFM orders have been shown in Table 2 . Ratio of FM order to AFM order $=12 / 73 \times 100 \% \approx 16 \%$. Calculation from the second assumption is more realistic. But the above calculations have been done in the most favourable case where at all the possible places of hole-pair formation, holes are present. In actual cases (from underdoped to optimum doped), there will be many patches where hole-pairs would not have been formed because no holes are available. Because of the small percentage of FM alignment in comparison to AFM alignment, the former may be submerged under AFM alignment in actual experiments. AFM coupling of spins of holes of $\mathrm{Cu}^{2+}$ spins is unable to explain superconductivity. But FM coupling of $\mathrm{Cu}^{2+}$ spins can explain superconductivity [4] but not supported by neutron scattering experiments. The reason may be that under overwhelming AFM order, minority transitory FM order is submerged. The present mechanism of hole-pair formation is supported by theoretical considerations [4] and cannot be rejected by experiments because of its indetectibility of FM order due to its lean and fleeting presence. Time-period of a hole in Y-123 is $10^{-13}$ sec. And in this time a hole has to cross 10 cells (not only 9 cells but 10 cells because $\mathrm{C}$ cell will come twice in its path). Time spent in $\mathrm{C}$ cell will be greater than in any other cell, because in this cell both of the holes face Coulomb repulsion and their velocities are reduced and practically there is no magnetic order in this cell. So a hole has to pass through 8 cells only with some kind of FM order and it will take less than $10^{-14} \mathrm{sec}$ per cell. There is another reason for FM order not to be observed in experiments is that half of time, projections of holes will be above the plane and half of time below the plane. They may cancel each other because changeover is very fast. It may be possible 
that the transitory FM order in such a shot spell is not detected in neutron scattering experiments. Kopp et al. [28] and Sonier et al. [29] have maintained that in superconductivity dome in the phase diagram, FM order coexists with AFM order.

Formation of hole-pairs in cuprates with large coherence lengths in a-b plane $\xi_{a b}$ : We have till now discussed formation of preformed hole-pairs taking Y-123 as a representative example which has coherence length $\xi_{a b}$ of the order of $15-20 \AA$. But there are cuprate superconductors whose $\xi_{a b}$ are much larger than that of Y-123. There are also cuprates whose $\xi_{a b}$ are nearly equal to that of Y-123 or a little smaller. They can be understood on the lines of arguments given for Y-123. The problem is how to explain hole-formation when $\xi_{a b}$ is quite large. A broad view of the properties of cuprate superconductors is given in the table 3 with the parameters with which we may be concerned in this paper. From Table 3, one thing becomes clear that for a single $\mathrm{CuO}_{2}$ layered superconductor, $\xi_{a b}$ has the largest value, followed by two layered and the smallest ones are for the three layered cuprates. Coherence lengths along c-axis $\xi_{c}$ are quite small in all cases. For small $\xi_{c}$ values, Kumar et al. [30] have given a reasonable explanation for highly enhanced resistivity in c-axis transport in normal state. They interpreted the suppression of single particle transport along the c-axis in the normal state due to the blocking of inter-block transport by the intra-block coupling to many-body environments (i.e., entanglement with other electronic degrees of freedom). This mechanism is called Quantum Zeno Effect (QZE). From this it can be said that coherence lengths depend on the velocity of transport of charge carriers. Regarding the values of $\xi_{a b}$ it can be said that when there are more than one $\mathrm{CuO}_{2}$ layer, there is some kind of interaction between layers that reduces the velocity of charge carriers which lowers the value of $\xi_{a b}$ in two $\mathrm{CuO}_{2}$ layered and the lowest value in three layered cuprates. Example of interaction between layers of $\mathrm{CuO}_{2}$ in cuprate superconduc-

Table 2. Showing positions of holes in different $(\mathrm{CuO})_{4}$ plaquette or cells, no. of cells in FM or half FM coupling and no. of cells in AFM coupling.

\begin{tabular}{cccc}
\hline S. No. & & No. of cells in FM and half-AFM couplings & Positions of holes in cells, first Hole 1 and then 2 \\
\hline 1 & A, E & FM = 2 & 7 \\
2 & B, D & Half - FM $=4=2 \mathrm{FM}$ & 5 \\
3 & C, C & No coupling & 7 \\
4 & H, G & Half - FM $=2=1$ FM & 7 \\
5 & I, F & FM = 2 & 5 \\
6 & H, G & Half - FM $=4=2$ FM & 8 \\
7 & C, C & No coupling & 7 \\
8 & D, B & Half - FM $=2=1$ FM & 7 \\
9 & E, A & FM $=2$ & AFM $=61$ \\
\hline
\end{tabular}

Table 3. Showing for different superconductors crystal structure, coherence lengths in ab-plane and along c-axis in Angstroms, energy of odd and even inelastic neutron neutron scattering peaks in milielectron volts, superconducting energy gap $(2 \Delta)$ and ratio of $(2 \Delta)$ and energy of odd peak ( $2 \Delta /$ Eodd).

\begin{tabular}{|c|c|c|c|c|c|c|c|c|}
\hline Substances & $\begin{array}{l}\text { Crystal Structure } \\
\qquad\left(A^{\circ}\right)\end{array}$ & $\begin{array}{c}T_{c} \\
(K)\end{array}$ & $\xi_{a b} \quad\left(A^{\circ}\right)$ & $\xi_{c}\left(A^{\circ}\right)$ & $\begin{array}{c}\text { Energy of odd } \\
\text { peak (meV) }\end{array}$ & $\begin{array}{l}\text { Energy of } \\
\text { even peak } \\
\text { (meV) }\end{array}$ & $2 \Delta \quad(\mathrm{meV})$ & $2 \Delta / E o$ dd \\
\hline La-214 & $\mathrm{a}=\mathrm{b}=3.77, \mathrm{c}=13.25$ & 38 & 21 & - & 18 & - & 35 & \\
\hline $\mathrm{Y}-123$ & $\mathrm{a}=3.823, \mathrm{~b}=3.885, \mathrm{c}=11.7$ & 92 & $13-16$ & $2-3$ & 41 & 53 & 79 & \\
\hline $\mathrm{Bi}-2212$ & $\mathrm{a}=5.40, \mathrm{~b}=5.41, \mathrm{c}=37.01$ & 96 & $27-38$ & $1.6-1.8$ & 43 & - & - & - \\
\hline Bi-2223 & $\mathrm{a}=5.42, \mathrm{~b}=5.41, \mathrm{c}=37.01$ & 122 & 29 & 0.93 & - & - & - & - \\
\hline Tl-2201 & $\mathrm{a}=\mathrm{b}=3.85, \mathrm{c}=23.2$ & 92 & 52 & 3 & 47 & - & 85 & 1.81 \\
\hline Tl-2212 & $\mathrm{a}=\mathrm{b}=3.85, \mathrm{c}=23.2$ & 118 & $20-31$ & $0.3-6.8$ & - & - & - & - \\
\hline Tl-2223 & $\mathrm{a}=\mathrm{b}=3.85, \mathrm{c}=35.6$ & 128 & $11-13.6$ & - & - & $=$ & - & - \\
\hline Hg-1201 & $\mathrm{a}=\mathrm{b}=3.85, \mathrm{c}=9.5$ & 95 & $17-34$ & - & 56 & - & 88 & 1.57 \\
\hline Hg-1212 & - & 127 & 21.1 & - & - & - & - & - \\
\hline Hg-1223 & $\mathrm{a}=\mathrm{b}=3.857, \mathrm{c}=15.7$ & 133 & 13 & - & 72 & - & 117 & 1.63 \\
\hline
\end{tabular}


tors is the occurrence of odd and even AFM excitations in two $\mathrm{CuO}_{2}$ layered cuprates. It explains why $\xi a b$ is highest in single layered cuprates. As in multi-layered cuprates, in single layered cuprates also velocity of holes depends on the buckling of $\mathrm{Cu}-\mathrm{O}-\mathrm{Cu}$ angle in the $\mathrm{CuO}_{2}$ plane. Buckling may arise due to interaction of $3 d_{z 2-r 2}$ and $2 p_{z}$ orbitals of the oxygen ions of nearest layers vertically above or below. Interaction between $3 d_{x 2-y 2}$ orbital of the $\mathrm{Cu}^{2+}$ ion and $2 p x$ and $2 p y$ orbitals of the planar oxygen when a hole reaches $\mathrm{O}^{2-}$ ion may be another cause of buckling.

Chmaisen et al. [31], observed that for a fixed chemical composition, increased $\mathrm{CuO}_{2}$ plane buckling lowers the transition temperature. Amit Keren [32] while investigating superconductor $\left(\left\{\mathrm{Ca}_{\mathrm{x}} \mathrm{La}_{1-\mathrm{x}}\right\} \mathrm{Ba}_{1.75-\mathrm{x}} \mathrm{La}_{0.25 \mathrm{x}}\right)$ concluded that $T_{c}$ is determined by a single scale set by super exchange interaction $J$, which in turn is determined by the in-plane $\mathrm{Cu}-\mathrm{O}-\mathrm{Cu}$ buckling angle. Thus buckling angle affects $T_{c}$, super exchange interaction and also coherence length.

As seen from Table 3, the in-plane coherence length $\xi_{a b}$ in Tl-2201 is equal to $52 \AA$ (highest in cuprates). It means that in the beginning of hole-pair formation, two holes are situated at a distance of $52 \AA$ which is equivalent to $\approx 13(\mathrm{CuO})_{4}$ plaquettes. For hole-pair formation the wave function of Hole 1 in the first cell (in a figure similar to Figure 1 but with 13 cells in y-direction and 13 cells in the $\mathrm{x}$-direction intersecting each other at the central 7th cell) must overlap with that of Hole 2 in the 13th cell. The odd resonance peak in this superconductor has been found to be equal to $47 \mathrm{meV}$, which when equated to $\mathrm{h} v$ gives $v=1.135 \times 1013$ hertz or time-period $T=8.81 \times 10^{-14}$ second. As has been shown in [4] that for hole-pair formation in Y-123, each hole has to cover a distance of $\approx 38.5 \AA$ (equivalent to 10 cell length) in one time-period $=10^{-13}$ sec. For hole-formation in Tl-2201, each hole has to cover a distance of $\approx 100 \AA$ (equivalent to 26 cell length) in one time-period $=8.81 \times 10^{-14}$ second.

It has been found that $\mathrm{CuO}_{2}$ plane in TL-2201 is quite flat and smooth. The effect of smoothness of $\mathrm{CuO}_{2}$ plane can also be guessed from comparison of the velocities of hole pairs in Y-123 and Tl-2201. In Y-123, each hole of a hole-pair covers angular distance $\omega t=360^{\circ}$ in $10^{-13} \mathrm{sec}$ corresponding to linear distance of $10 \times 3.84=38.4 \AA$ (each side of the cell taken to be equal to $38.4 \AA$ ). In the case of Tl-2201, the linear distance $=26 \times 3.84=99.84 \AA$. Velocity of hole-pair = distance travelled/time period. For Y-123, velocity $=\left(38.4 \times 10^{-8}\right) / 10^{-13}=3.84 \times 10^{6} \mathrm{~cm} / \mathrm{sec}$.

For Tl-2201, velocity $=\left(99.84 \times 10^{-8}\right) / 8.81 \times 10^{-14}=11.33 \times 10^{6} \mathrm{~cm} / \mathrm{sec}$. Thus velocity of hole pairs in Tl2201 is $\approx 2.95$ times more than that in $Y$-123. It is known that velocity of hole-pairs in cuprates is proportional to $n_{s} / m^{*}$ (where $n_{s}$ superconducting density and $m^{*}=$ effective mass of hole pair). All the data about Y123 and Tl-2201 used here have been taken for optimum doping, hence $\mathrm{n}_{\mathrm{s}}$ will be nearly equal in both cases. So velocity of hole-pairs should be proportional to $1 / \mathrm{m}^{*}$. Thus it can be concluded that the coherence length of cuprates depends upon the effective mass of hole-pair in superconductors. It may also be mentioned here that effective mass of hole-pairs is less than that of single hole.

Different effective masses of hole-pairs in different cuprates may be attributed to the nature of near neighbours of $\mathrm{CuO}_{2}$ plane in the vertical direction. In Y-123, near neighbours are as follows: $\mathrm{CuO}-\mathrm{BaO}-\mathrm{CuO}_{2}-\mathrm{Y}-$ $\mathrm{CuO}_{2}-\mathrm{BaO}-\mathrm{CuO}$ and for Tl-2201, they are as follows: TlO-TlO-BaO-CuO${ }_{2}-\mathrm{BaO}-\mathrm{TlO}-\mathrm{TlO}$. Near neighbours in the two cases are quite different.

Near neighbours control the smoothness and flatness of the plane or buckling of $\mathrm{Cu}-\mathrm{O}-\mathrm{Cu}$ angle which ultimately controls the velocity of holes in the $\mathrm{CuO}_{2}$ plane. One difference that is obvious in the immediate neighbourhood of $\mathrm{CuO}_{2}$ plane in Tl-2201 and Y-123 is that in the former, $\mathrm{Cu}^{2+}$ ions of $\mathrm{CuO}_{2}$ plane have bipyramidal coordinations with the $\mathrm{O}^{2-}$ ions of $\mathrm{BaO}$ plane, whereas in the latter, on one side of $\mathrm{CuO}_{2}$ plane , there is coordination between $\mathrm{Cu}^{2+}$ ions of $\mathrm{CuO}_{2}$ plane with $\mathrm{O}^{2-}$ ions of $\mathrm{BaO}$ plane but on the other side, $\mathrm{Cu}^{2+}$ ions of $\mathrm{CuO}_{2}$ plane are not coordinated to any oxygen ion , because in $\mathrm{Y}$ layer there is no oxygen ion. In addition to coordination of ions, the atoms of surroundings may affect the properties of $\mathrm{CuO}_{2}$ layers by differences in electronic structure, ionization energy and electronegativity etc.

The general condition for Heisenberg exchange interaction to take place is that the ratio $r_{i j} / r_{3 d}$ must be greater than 3 but not much greater, where $r_{i j}=$ distance between atoms $i$ and $j$ atoms and $r_{3 d}=$ radius of the $3 \mathrm{~d}$ orbital. But there are instances where Heisenberg exchange interaction is effective at much larger separations. In a series of free radicals in aqueous solution, the exchange rate [33] is much greater than the reaction rate and the critical exchange distance is between one and three hard sphere encounter distance in agreement with several theoretical predictions. In [33], exchange interaction has been found to be effective at distances $\approx 30 \AA$. Velocity of a hole in Tl-2201 is much higher than that in Y-123. Higher velocity means that the surface is flat and 
smooth which means that there is no or very small variation of electron density along its path or the buckling angle is zero or very small. When intervening space between two holes is smooth or without any variation of electron density, the wave functions of electrons can spread over large distances. Holes situated at much larger distances than $r_{i j} / r_{3 d}$ can also be bound by Heisenberg exchange interaction. It can be concluded that hole-pair formation in Tl-2201 (with very large $\xi_{a b}$ ) and Y-123 (with small or moderate $\xi_{a b}$ ) can be explained on the same lines by transitory FM order induced by holes wandering in the $\mathrm{CuO}_{2}$ plane.

\section{Summary}

Following are the important points in this paper:

1) We briefly described our EPR work on deoxygenated cuprate superconductors. It was inferred from our work that an isolated $(\mathrm{CuO})_{4}$ plaquette (after breaking of all its $8 \mathrm{Cu}-\mathrm{O}$ bonds at its 4 corners from the surrounding) is equivalent to a $(\mathrm{CuO})_{4}$ plaquette of continuous $\mathrm{CuO}_{2}$ plane with a hole inside it. In isolated $(\mathrm{CuO})_{4}$ plaquettes, magnetic field is generated due to the alignment of spins of $4 \mathrm{Cu}^{2+}$ ions in the plaquettes. So it was concluded that a hole on entering a $(\mathrm{CuO})_{4}$ plaquette of continuous $\mathrm{CuO}_{2}$ sheet will also produce magnetic field caused by the alignment of spins of $4 \mathrm{Cu}^{2+}$ holes.

2) When a hole proceeds along a column or row of plaquettes in a continuous $\mathrm{CuO}_{2}$ plane, magnetic field produced goes on oscillating from a direction vertically upward of the $\mathrm{CuO}_{2}$ plane to vertically downward, attaining zero while crossing the plane. Also the magnitude of the magnetic field goes on changing when holes pass from one cell to the other. When two holes proceeding towards each other along a column or row of cells in $\mathrm{CuO}_{2}$ plane come at a certain separation between them, are attracted towards each other by Heisenberg exchange interaction. But the direction of their motion is decided by the magnitude and direction of magnetic field produced when holes move from one cell to the other. Two holes moving along $\mathrm{CuO}_{2}$ plane under the effect of exchange interaction and magnetic field form a hole-pair and they carve out a path which is similar to $3 d_{x 2-y 2}$ atomic orbital. For hole-pair formation, Y-123 has been taken as an example.

3) In the above model, no magnetic field was supposed to be present in any cell, until a hole enters a cell. The above model was extended to the case when AFM alignment of spins is present in all the cells except those cells where FM alignment is present due to entry of holes. Again example was Y-123.

4) The model proposed in [4] for Y-123 with planar coherence length $=15-20 \AA$ in a-b plane has been extended to Tl-2201 with very high coherence length $=52 \AA$. The model seems to be successful even in Tl-2201. The model seems to be successful because of the following two reasons. The $\mathrm{CuO}_{2}$ plane in $\mathrm{Tl}-2201$ is more flat and smooth. Due to the smoothness of the path between two holes, the velocity of hole-pairs in Tl-2201 is nearly 2.95 times more than that in $\mathrm{Y}-123$.

5) Flatness or smoothness of the $\mathrm{CuO}_{2}$ plane is attributed to the nature of near neighbours of $\mathrm{CuO}_{2}$ plane in the superconductor. Differences between the near neighbours of $\mathrm{CuO}_{2}$ plane in Y-123 and $\mathrm{Tl}-2201$ have been recounted.

\section{References}

[1] Dai, P.C., Mook, H.A., Aeppli, G., Hayden, S.M. and Dogan, F. (2000) Resonance as a Measure of Pairing Correlations in High $\mathrm{T}_{\mathrm{c}}$ Superconductor $\mathrm{YBa}_{2} \mathrm{Cu}_{3} \mathrm{O}_{6.6}$. Nature, 406, 965-968. http://dx.doi.org/10.1038/35023094

[2] Stankowski, J., Krupski, M. and Roman, M. (2004) Remarks on the Phase Diagram of High Temperature Superconductors. Materials Science-Poland, 22, 175.

[3] Singh, R.J. (2009) Preformed Hole-Pairs in Cuprate Superconductors. International Journal of Modern Physics B, 231, 53-76. http://dx.doi.org/10.1142/S0217979209049590

[4] Singh, R.J. (2011) Model of Preformed Hole-Pairs in Cuprate Superconductors. Journal of Modern Physics, 2, 885-897. http://dx.doi.org/10.4236/jmp.2011.28105

[5] Singh, R.J. (2012) Model of Preformed Hole-Pairs in c-Axis Transport in Cuprate Superconductors. World Journal of Condensed Matter Physics, 2, 228-236.

[6] Punnoose, A., Maurya, B.P., Jilson, M., Umar, M., Haque, M.I. and Singh, R.J. (1993) EPR Observation of $\mathrm{Cu}^{2+}-\mathrm{Cu}^{2+}$ Pairs in Cupric Oxide Powder. Solid State Communications, 88, 195-198. http://dx.doi.org/10.1016/0038-1098(93)90740-E

[7] Singh, R.J., Alex, P., Mathew, J., Maurya, B.P. and Khan, S. (1994) S = 1 and S = 2 EPR Signals in Modified CuO and $\mathrm{BaCuO}_{2}$. Physical Review B, 49, 1346. http://dx.doi.org/10.1103/PhysRevB.49.1346 
[8] Singh, R.J., Ikram, M., Singh, A., Punnoose, A., Maurya, B.P. and Shakeel, K. (1995) Copper Tetramers in High Temperature Superconductors. Physics Letters A, 208, 369-374. http://dx.doi.org/10.1016/0375-9601(95)00674-8

[9] Punnoose, A. and Singh, R.J. (1995) EPR Studies of High $\mathrm{T}_{\mathrm{c}}$ Superconductors and Related Systems. International Journal of Modern Physics B, 9, 1123. http://dx.doi.org/10.1142/S0217979295000471

[10] Singh, R.J., Sharma, P.K., Singh, A. and Shakeel, K. (2001) EPR Spectra of Deoxygenated High Temperature Superconductors. Physica C, 356, 285-296. http://dx.doi.org/10.1016/S0921-4534(01)00283-0

[11] Khan, S., Mohd, I., Arti, S. and Singh, R.J. (1997) EPR Study of Deoxygenated $\mathrm{La}_{2} \mathrm{CuO}_{4}$. Physica C, 281, $143-148$. http://dx.doi.org/10.1016/S0921-4534(97)00328-6

[12] Shakeel, K., Arti, S. and Singh, R.J. (1998) EPR Study of $\mathrm{La}_{2-\mathrm{x}} \mathrm{M}_{\mathrm{x}} \mathrm{CuO}_{4}(\mathrm{M}=\mathrm{Sr}$, Ba). Solid State Communications, 106, 621-626. http://dx.doi.org/10.1016/S0038-1098(98)00101-X

[13] Shakeel, K., Arti, S. and Singh, R.J. (1999) EPR Study of $\mathrm{La}_{1.854} \mathrm{Sr}_{0.146} \mathrm{CuO}_{4}$. Physica C: Superconductivity, 325, 165-172. http://dx.doi.org/10.1016/S0921-4534(99)00513-4

[14] Meng, Q.B., Wu, Z.J. and Zhang, S.Y. (1998) Evaluation of the Energy Barrier Distribution in Many Particle Systems Using the Path Integral Approach. Journal of Physics: Condensed Matter, 10, L89.

[15] Rossat-Mignod, J., Regnault, L.P., Veltier, C., Bourges, P., Burtlet, P., Bossy, J., Henry, J.Y. and Caperlot, G. (1991) Neutron Scattering Study of $\mathrm{YBa}_{2} \mathrm{Cu}_{3} \mathrm{O}_{6+\mathrm{x}}$ System. Physica C, 185-189, 86-92. http://dx.doi.org/10.1016/0921-4534(91)91955-4

[16] Yu, G., Li, Y., Zhao, X., Barišić, N., Cho, Y., Bourges, P., Hradil, K., Mole, R.A. and Greven, M. (2010) Magnetic Resonance in the Model High-Temperature Superconductor $\mathrm{HgBa}_{2} \mathrm{CuO}_{4+\delta}$. Physical Review B, 81, Article No: 064518. http://dx.doi.org/10.1103/PhysRevB.81.064518

[17] Pailhès, S., Ulrich, C., Faque, B., Hinkov, V., Sidis, Y., Ivanov, A., Lin, C.T., Keimerand, B. and Bourges, P. (2006) Doping Dependence of Bilayer Resonant Spin Excitations in (Y, $\mathrm{Ca}_{\text {Ba }} \mathrm{Ba}_{2} \mathrm{Cu}_{3} \mathrm{O}_{6+x}$. Physical Review Letters, 96, Article No: 257001. http://dx.doi.org/10.1103/PhysRevLett.96.257001

[18] Fong, H.F., Bourges, P., Sidis, Y., Regnault, L.P., Ivanov, A., Gu, G.D., Koshizuka, N. and Keimer, B. (1999) Neutron Scattering from Magnetic Excitations in $\mathrm{Bi}_{2} \mathrm{Sr}_{2} \mathrm{CaCu}_{2} \mathrm{O}_{8+\delta}$. Nature, 398, 588-591. http://dx.doi.org/10.1038/19255

[19] Capogna, L., Faque, B., Sidis, Y., Ulrich, C., Bourges, P., Pailhes, S., Ivanov, A., Tallon, J.L., Liang, B., Lin, C.T., Rykov, A.I. and Keimer, B. (2007) Odd and Even Magnetic Resonance Modes in Highly Overdoped $\mathrm{Bi}_{2} \mathrm{Sr}_{2} \mathrm{CaCu}_{2} \mathrm{O}_{8+\delta}$. Physical Review B, 75, Article No: 060502. http://dx.doi.org/10.1103/PhysRevB.75.060502

[20] He, H., Bourges, P., Sidis, Y., Ulrich, C., Regnault, L.P., Pailhes, S., Bazigirova, N.S. and Keimer, B. (2002) Magnetic Resonant Mode in the Single Layer High Temperature Superconductor $\mathrm{Tl}_{2} \mathrm{Ba}_{2} \mathrm{CuO}_{6+\delta}$. Science, 295, 1045-1047. http://dx.doi.org/10.1126/science.1067877

[21] Bourges, P., Regnault, L.P., Sidis, Y. and Veltier, C. (1996) Inelastic-Neutron-Scattering Study of Antiferromagnetic Fluctuations in $\mathrm{YBa}_{2} \mathrm{Cu}_{3} \mathrm{O}_{6.97}$. Physical Review B, 53, 876. http://dx.doi.org/10.1103/PhysRevB.53.876

[22] Batista, C.D., Ortiz, G. and Balasky, A.V. (2001) Unified Description of the Resonance Peak and Incommensuration in High- $\mathrm{T}_{\mathrm{c}}$ Superconductors. Physical Review B, 64, Article No: 172508. http://dx.doi.org/10.1103/PhysRevB.64.172508

[23] Sato, N.K., Aso, N., Miyake, K., Shiima, R., Thalmer, P., Varelogiannis, G., Geibel, C., Steglich, F., Fulde, P. and Komatsubara, T. (2001) Strong Coupling between Local Moments and Superconducting "Heavy" Electrons in $\mathrm{UPd}_{2} \mathrm{Al}_{3}$. Nature, 410, 340-343. http://dx.doi.org/10.1038/35066519

[24] Stock, C., Broholm, C., Hudis, J., Kang, H.J. and Petrovic, C. (2008) Spin Resonance in the $d$-Wave Superconductor CeCoIn ${ }_{5}$. Physical Review Letters, 100, Article No: 087001. http://dx.doi.org/10.1103/PhysRevLett.100.087001

[25] Christiansen, A.D., McMorrow, D.F., Rønnow, H.M., Lake, B., Heiden, S.M., Aeppli, G., Perring, T.G., Mangkorntong, M., Nohara, M. and Takagi, H. (2008) Dispersive Excitations in the High Temperature Superconductor $\mathrm{La}_{2-\mathrm{x}} \mathrm{Sr}_{\mathrm{x}} \mathrm{CuO}_{4}$. Nature, 456, 930.

[26] Yu, G., Li, Y., Motoyama, E.M. and Greven, M. (2009) A Universal Relationship between Magnetic Resonance and Superconductivity Gap in Unconventional Superconductors. Nature Physics, 5, 873-875. http://dx.doi.org/10.1038/nphys1426

[27] McDonald, R.D., Harrison, N. and Singleton, J. (2009) Exact Mapping of the $d_{x 2-y 2}$ Cooper-Pair Wavefunction onto the Spin Fluctuations of the Cuprate: The Fermi Surface as a Driver for "High $\mathrm{T}_{\mathrm{c}}$ " Superconductivity. Journal of Physics: Condensed Matter, 21, Article No: 012201.

[28] Angela, K., Amit, G. and Sudip, C. (2007) Competing Ferromagnetism in High Temperature Copper Oxide Superconductors. Proceedings of the National Academy of Sciences of the United States of America, 104, 6123-6127.

[29] Sonier, J.E., Kaiser, C.V., Pacradoum, V., Sabok-Sayer, S.A., Cochrane, C., MacLaughlin, D.E., Komaya, S. and Hussey, N.E. (2010) Direct Search for a Ferromagnetic Phase in a Heavily Overdoped Nonsuperconducting Copper Oxide. 
Proceedings of the National Academy of Sciences of the United States of America, 107, 17131-17134.

[30] Kumar, N. and Jayannavar, A.M. (1992) Temperature Dependence of $c$-Axis Resistivity of High- $\mathrm{T}_{\mathrm{c}}$ Layered Oxides. Physical Review B, 45, 5001. http://dx.doi.org/10.1103/PhysRevB.45.5001

[31] Chmaisen, O., Jorgensen, J.D., Short, S., Knizhnik, A., Eikstein, Y. and Shaked, H. (1999) Scaling of Transition Temperature and $\mathrm{CuO}_{2}$ Plane Buckling in High Temperature Superconductors. Nature, 397, 45-48. http://dx.doi.org/10.1038/16209

[32] Keren, A. (2009) Evidence for Magnetic Mechanism for Cuprate Superconductivity. New Journal of Physics, 11, Article No: 065006. http://dx.doi.org/10.1088/1367-2630/11/6/065006

[33] Bartels, D.M., Trifunac, A.D. and Lawler, R.G. (1988) Observation of Heisenberg Spin Exchange between Reactive Free Radicals. Chemical Physics Letters, 152, 109-115. http://dx.doi.org/10.1016/0009-2614(88)87337-8 
Scientific Research Publishing (SCIRP) is one of the largest Open Access journal publishers. It is currently publishing more than 200 open access, online, peer-reviewed journals covering a wide range of academic disciplines. SCIRP serves the worldwide academic communities and contributes to the progress and application of science with its publication.

Other selected journals from SCIRP are listed as below. Submit your manuscript to us via either submit@scirp.org or Online Submission Portal.
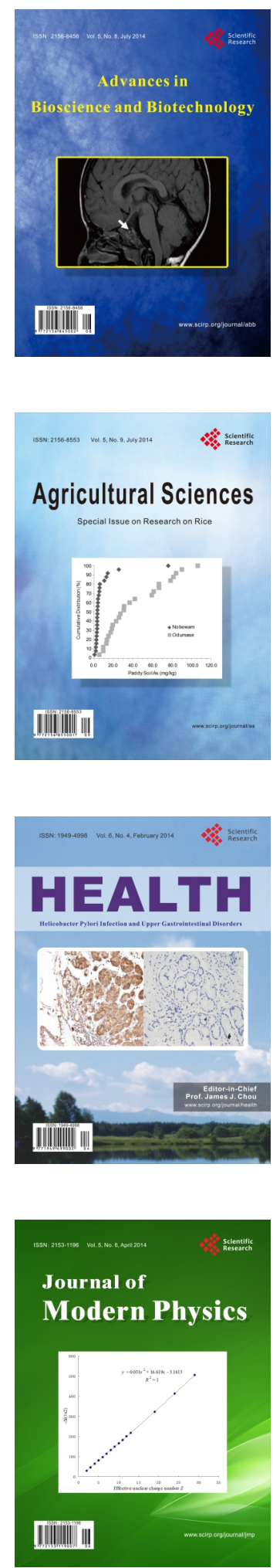
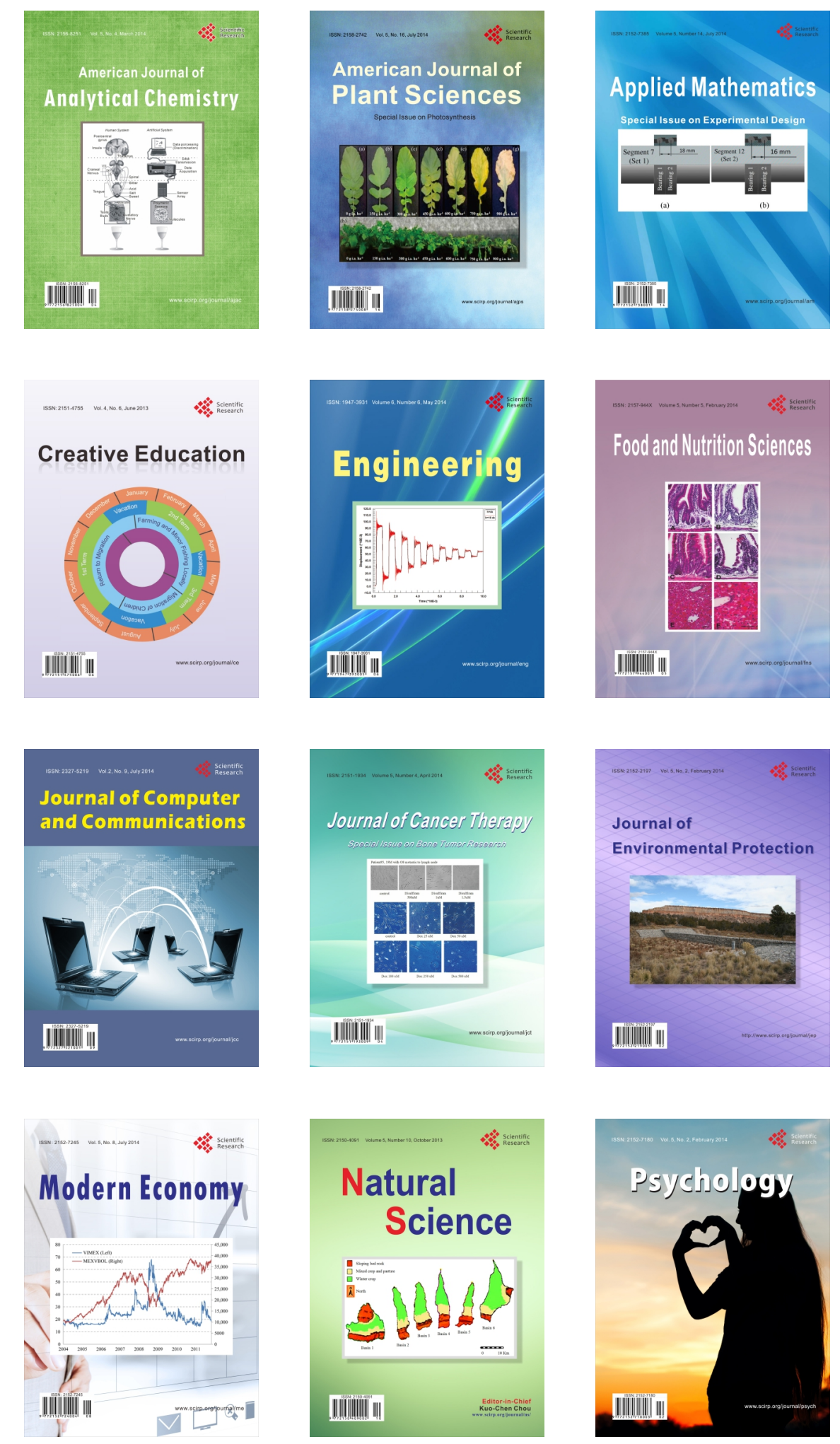